\title{
Viabilidade financeira de instalação de uma unidade de beneficiamento de cascas de coco verde
}

Financial feasibility of installing a processing unit of green coconut shell

\author{
Vinícios Silveira" $^{1 *}$, Thiago Ricielli de Paula Aragão² \\ 1 IPECEGE - Especialista em Agronegócios - Av. Neiva Moreira, 400 - Bairro Calhau - CEP 65071-383 - \\ São Luís (MA), Brasil \\ 2 ESALQ/USP - Doutorando em Genética e Melhoramento de Plantas - Av. Pádua Dias, 11 - Bairro \\ Agronomia - CEP 13418-900 - Piracicaba (SP), Brasil
}

\section{Resumo}

A produção nacional de coco (Cocos nucifera) destinada para o consumo de água in natura e industrializada no ano de 2014 foi de aproximadamente 1,5 bilhão de frutos. Toneladas de cascas foram, em sua maioria, descartadas nos lixões e aterros sanitários das cidades, desprezando, dessa forma, uma matéria prima potencial de alta aplicabilidade e baixo custo. $O$ presente trabalho tem o objetivo de apurar a viabilidade financeira a partir do levantamento dos custos de instalação de uma unidade de beneficiamento de cascas de coco verde para produção de pó e fibras brutas. A unidade será implantada no município de São Luís (MA). Os quesitos técnicos utilizados como referência foram desenvolvidos e testados pela Empresa Brasileira de Pesquisa Agropecuária [Embrapa] Agroindústria Tropical. Para os cálculos de viabilidade foram utilizados, a partir do fluxo de caixa, os critérios financeiros valor presente líquido [VPL], payback descontado e taxa interna de retorno [TIR], além de análise de sensibilidade de cenários. O maior volume de capital destinou-se às instalações físicas da unidade, consumindo $R \$ 550.415,00$, enquanto as máquinas e equipamentos tiveram dispêndios de $R \$ 284.650,00$. O projeto é financeiramente atrativo, gerando VPL de $R \$ 361.458,00$ e TIR de $16 \%$, frente a uma taxa mínima de atratividade de $7,13 \%$, ao passo que os recursos investidos serão retornados no sexto ano. O projeto é sensível às variações de preço dos produtos vendidos.

Palavras-chave: Cocos nucifera, projeto de investimento, valor presente líquido

\begin{abstract}
The national coconut (Cocos nucifera) production for natural and industrialized water, in 2014, was approximately 1.5 billion fruits. Tons of shells was mostly discarded in dumps and landfills, undervaluing a raw material with potential applicability and low cost. This study aims to determine the financial viability of a coconut shell processing installation for coconut shells powder production and husk fiber in São Luís - Maranhão State. The Brazilian Agricultural Research Corporation [Embrapa] Agroindústria Tropical tested and developed the technical requirements used as reference. To calculate the financial viability, it was used the concepts of net present value [NPV], discounted payback period, internal rate of return [IRR] besides a sensitivity analysis of scenarios. The physical facilities of the unit allocated the largest amount of the capital, consuming $R \$ 550,415.00$, while machinery and equipment expenditures were $R \$ 284,650.00$. The project is financially attractive due to an NPV of $R \$ 361,458.00$ and IRR of $16 \%$ considering a hurdle rate of $7.13 \%$, while the resources invested would be returned within approximately six years. The project is sensitive to price variation of the goods sold.
\end{abstract}

Keywords: Cocos nucifera, investment project, net present value

\footnotetext{
${ }^{1}$ Autor correspondente <souvinicios@icloud.com>

Enviado: 31 maio 2016

Aprovado: 30 jun. 2016
} 


\section{Introdução}

O coqueiro (Cocos nucifera) é uma das plantas que melhor se disseminou pelo mundo, notadamente em regiões de clima semelhante ao encontrado em sua região originária, o Sudeste Asiático (Foale e Harries, 2009). Condições climáticas favoráveis ao seu desenvolvimento são observadas principalmente entre as latitudes $20^{\circ} \mathrm{N}$ e $20^{\circ}$ $\mathrm{S}$, demonstrando assim, sua preferência por climas tropicais e subtropicais (Cuenca, 2007).

De acordo com a Food and Agriculture Organization of the United Nations [FAO] (2013), a produção mundial de coco no ano de 2013 foi de 55 milhões de toneladas em área colhida de 12 milhões de hectares. Os cinco maiores países produtores concentraram conjuntamente $92 \%$ do total produzido, com destaque individual para a Indonésia, que produziu 18 milhões de toneladas (33\%), numa área de aproximadamente três milhões de hectares. O Brasil aparece na quarta posição com $5 \%$ da produção mundial, ou três milhões de toneladas, em uma área de aproximadamente 257 mil hectares.

No âmbito nacional a produção é calculada em quantidade de frutos produzidos, diferentemente do apresentado, onde a FAO contabiliza os resultados em toneladas. Conforme previsão do Sindicato Nacional dos Produtores de Coco do Brasil [Sindcoco] (2014), a produção destinada para venda de produtos como água de coco industrializada e para o consumo in natura deve atingir cerca de 1,5 bilhão de frutos no ano de 2014. Ainda em se tratando do cenário brasileiro, a produção total de coco atingiu 1,9 bilhão de frutos no ano de 2012, gerando receitas de $R \$ 898$ milhões numa área colhida de 257 mil hectares (IBGE, 2014). Do ponto de vista econômico, a Região Nordeste possui grande importância e liderança de mercado, sendo responsável por $65 \%$ das riquezas geradas na cadeia desse agronegócio, com produção de 1,3 bilhão de frutos no ano de 2012 (IBGE, 2014).

Segundo Cuenca (2007), a produção brasileira de coco destina-se, em grande parte, à agroindústria para produção de coco ralado e leite de coco, visando atender a demanda de grandes empresas produtoras de doces, biscoitos e chocolates. Cerca de $70 \%$ da produção é consumida nesse processo. Ainda de acordo com a instituição, os $30 \%$ restantes da produção ficam no mercado nordestino, onde $20 \%$ são consumidos na forma de coco verde.

O coco verde é um produto volumoso, pesando em média $1,5 \mathrm{~kg}$, sendo que $80 \%$ do seu peso corresponde às cascas e os $20 \%$ restantes à água (Cuenca, 2007). 
Dessa forma, pode-se estimar que no Nordeste sejam geradas anualmente 312 mil toneladas de cascas de coco verde somente com o consumo do fruto in natura.

A indústria de processamento de coco já utiliza amplamente as cascas do fruto maduro como combustível para suas caldeiras ou no processamento para obtenção de fibras longas ("coir"), que são utilizadas para manufatura de cordas, mantas, tapetes e muitos outros produtos. Segundo a FAO (2013), a produção mundial de fibra de coco ou "coir", alcançou 1,09 milhão de toneladas, com destaque para a Índia, que produziu $47 \%$ do total, ou 514 mil toneladas no ano de 2012.

O processo de extração das fibras gera como subproduto o pó ("coir pith" ou "coir dust"). Esse dejeto já é amplamente utilizado como base para formulação de substrato agrícola natural em diversos países do mundo. Porém ainda são poucos os estudos sobre as formas de se beneficiar e aproveitar as cascas do coco verde para uso comercial de suas fibras e do pó no Brasil, isso muito em função das características físico-químicas desse produto, tais como a alta concentração de sais, taninos, umidade, volume e peso.

Nesse sentido, a Embrapa Agroindústria Tropical lançou um estudo detalhado sobre uma nova tecnologia desenvolvida por seus pesquisadores para processamento das cascas de coco verde. Em parceria com uma metalúrgica do Ceará, foi desenvolvido um conjunto de máquinas e equipamentos para viabilizar o processo mecanizado e automatizado para trituração das cascas, extração da umidade, dos excessos de sais e da separação das fibras do pó (Mattos et al., 2011).

Assim, frente ao potencial de utilização da grande quantidade de cascas de coco verde gerada, o presente trabalho tem como objetivo avaliar do ponto de vista financeiro a viabilidade de instalação de uma unidade de beneficiamento dessa matéria prima, ainda pouco utilizada, de baixo custo e de alta aplicabilidade, uma vez que o Nordeste brasileiro possui potencial para exploração em grande escala desse mercado, principalmente quando avaliados os atuais preços praticados no mercado externo para venda desses produtos.

\section{Materiais e Métodos}

\section{Cidade alvo do estudo}

Para este trabalho utilizou-se a cidade de São Luís - capital do estado do Maranhão, região Nordeste do Brasil - como referência para elaboração dos cálculos de viabilidade financeira de instalação da unidade de processamento de cascas de coco verde. Segundo estimativas do Instituto Brasileiro de Geografia e Estatística [IBGE] 
(2014), a cidade possuía 1,064 milhão de habitantes em 2014. Porém, atualmente não existem aterros sanitários que atendam a região metropolitana da cidade, tampouco conhece-se iniciativas para descarte adequado dos resíduos produzidos pelo consumo da água de coco nas praias e nos bairros da cidade.

Segundo levantamento realizado neste trabalho diretamente com os principais atacadistas da cidade, o consumo anual do fruto atingiu cerca de seis milhões de unidades no ano de 2013, sendo que para suprir o consumo interno os distribuidores locais precisaram recorrer à importação de frutos de outros estados do Nordeste, pois segundo o último levantamento do IBGE (2014) a produção local (Ilha de São Luís e cidade de Santa Rita) não passou de 358 mil unidades em 2013.

Com base no relato de Cuenca (2007), de que $80 \%$ do peso do coco verde refere-se à sua casca, pode-se estimar em 1,2 kg a quantidade de resíduos sólidos produzidos por cada fruto consumido. Portanto, estimou-se que a geração de lixo resultante do consumo do coco verde alcançou aproximadamente 7.200 toneladas anuais somente na cidade alvo do estudo. Esse volume de matéria prima seria mais do que suficiente para suportar a necessidade mínima de processamento exigida pelos parâmetros técnicos da unidade de beneficiamento simulada.

Diante das características e do cenário apresentado, fica notória a potencialidade que a cidade escolhida possui para implementação de uma unidade de processamento de cascas de coco verde.

\section{Aspectos técnicos do processamento}

Utilizou-se como parâmetro de processamento médio a quantidade de 1.200 $\operatorname{cocos} \mathrm{h}^{-1}$, quantidade essa que segundo a empresa fornecedora das máquinas necessárias para o processamento, está dentro do limite máximo suportado pelos equipamentos, que no caso são de $2.000 \operatorname{cocos~} \mathrm{h}^{-1}$. Multiplicando-se as horas trabalhadas diariamente, oito horas, pela capacidade de processamento e peso dos resíduos, $1,2 \mathrm{~kg}$ de casca, chegou-se a produção bruta de $11.520 \mathrm{~kg} \mathrm{dia}^{-1}$ de matéria processada. Aproximadamente $30 \%$ do peso da produção bruta diária referem-se à parte líquida das cascas do coco verde [LCCV], esse líquido é retirado ao final do processo de trituração e prensagem das cascas, reduzindo, dessa forma, a umidade das fibras, do pó e consequentemente o peso final da produção para $8.064 \mathrm{~kg} \mathrm{dia}^{-1}$.

De acordo com Mattos et al. (2014), após o processamento são gerados como subprodutos as fibras longas e o pó, que representam, respectivamente, 30\% e $70 \%$ da produção, ou $2.419 \mathrm{~kg} \mathrm{dia}^{-1}$ de fibras e $5.645 \mathrm{~kg} \mathrm{dia}^{-1}$ de pó. 
Segundo o Banco Central do Brasil [BACEN] (2014), o índice de utilização médio da capacidade instalada da indústria brasileira foi de $83,10 \%$. Ao aplicar-se esse índice na produção, obteve-se uma redução da produtividade para $2.010 \mathrm{~kg} \mathrm{dia}^{-1}$ de fibras e $4.691 \mathrm{~kg} \mathrm{dia}{ }^{-1}$ de pó.

A produção anual que será obtida em 256 dias de trabalhos de acordo com os parâmetros apresentados será, em valores arredondados, de 515 toneladas de fibras e 1.201 toneladas de pó.

\section{Investimentos}

Os investimos necessários para viabilizar a implementação da unidade foram divididos em dois grandes grupos: máquinas/equipamentos e obras/terreno. As máquinas serão produzidas pela metalúrgica parceira da Embrapa Agroindústria Tropical no desenvolvimento da tecnologia embarcada nos equipamentos base da usina de reciclagem (Mattos et al., 2011). Utilizou-se orçamento fornecido pela própria metalúrgica. $\mathrm{Na}$ Tabela 1 são apresentadas todas as máquinas, equipamentos e veículos necessários para o efetivo processamento das cascas de coco verde. O preço do secador tubular, da balança mecânica e da basculante, foi cotado por pesquisa de mercado. Já o valor do caminhão foi apurado pela tabela Fipe (2014), com o código do item 504135-0, para o mês de agosto de 2014.

Tabela 1. Máquinas e equipamentos necessários para implementação de uma unidade de processamento de cascas de coco verde em São Luís, Maranhão

\begin{tabular}{ccc}
\hline Item & Detalhamento & Valor \\
\hline Caminhão Ford & Ano 2014 & $-----\mathrm{R} \$-----$ \\
Prensa rotativa horizontal & Retira 80\% da umidade das cascas & $131.050,00$ \\
Classificador de fibra e pó & Separação da fibra e do pó & $25.950,00$ \\
Basculante de caminhão & Carroceria para transporte das cascas & $22.700,00$ \\
Secador tubular & Tipo contínuo para pó de coco & $20.000,00$ \\
Triturador de coco verde & Trituração das cascas & $17.800,00$ \\
Prensa hidráulica para 20 t & Prensa para produzir fardos de fibra & $17.600,00$ \\
Móveis e equipamentos & Uso do escritório & $13.000,00$ \\
Elevador sequencial & Transporte de cascas até o triturador & $7.900,00$ \\
Balança mecânica & Capacidade para 500 kg & $1.850,00$ \\
Máquina de costura industrial & Ensacar o pó das cascas & $1.800,00$ \\
\hline Total & & $284.650,00$ \\
\hline
\end{tabular}

Fonte: Elaborado pelo autor 
O espaço físico necessário para viabilizar a instalação da unidade foi definido em função da capacidade de processamento estabelecida. Os setores da unidade foram estruturados com base no estudo de Mattos et al. (2014), que define as dimensões necessárias para cada fase do processamento. Porém, verificou-se a necessidade de adaptação da estrutura para recepção de áreas administrativas, tais com: escritórios, refeitórios, vestiários, sala de qualidade de vida no trabalho e estacionamentos.

Optou-se pela aquisição de um terreno com tamanho mínimo para processamento diário de até 12.000 cocos, pois os preços praticados na cidade à época da pesquisa (set. 2014) mostraram-se vantajosos quando comparados com o custo de alugar-se espaço com a mesma dimensão. O orçamento das obras civis e o preço do terreno foram fornecidos por empresa especializada no ramo. Entre as obras a serem realizadas no terreno, estão a área administrativa, a moega de recepção das cacas (concreto simples), a área de tanques de alvenaria impermeabilizada para lavagem do pó, o pátio de secagem (concreto com juntas de dilatação), que será utilizado para secagem de fibras e pó, sendo que a cada 1.000 cocos são necessários $100 \mathrm{~m}^{2}$ (Tabela 2).

Tabela 2. Obras e terreno necessários para implementação de uma unidade de processamento de cascas de coco verde em São Luís, Maranhão

\begin{tabular}{|c|c|c|c|}
\hline Descrição & Quantidade & Valor Unitário & Valor Total \\
\hline & ------'m²----- & \multicolumn{2}{|c|}{ 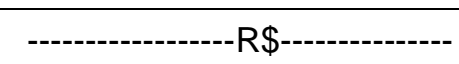 } \\
\hline Terreno & 2.023 & 80,00 & $161.840,00$ \\
\hline Estrutura coberta & 557 & 250,00 & $139.250,00$ \\
\hline Pátio de secagem & 1.200 & 110,00 & $132.000,00$ \\
\hline Área administrativa & 68 & $1.400,00$ & $95.200,00$ \\
\hline Estrutura coberta para estacionamento & 150 & 80,00 & $12.000,00$ \\
\hline Moega $^{(1)}$ & 40 & 174,00 & $6.960,00$ \\
\hline Área de tanques: profundidade de $1,2 \mathrm{~m}$ & 8 & 320,00 & $2.560,00$ \\
\hline Taxa da Prefeitura, CREA e Placa de Obra & - & - & 605,00 \\
\hline Total & & & $550.415,00$ \\
\hline
\end{tabular}

Nota: (1) Moega com $5 \mathrm{~m}$ de largura, $1 \mathrm{~m}$ de altura e $8 \mathrm{~m}$ de comprimento Fonte: Elaborado pelo autor

\section{Despesas e custos anuais}

Estimou-se em 20 o número de funcionários necessários para operacionalizar a unidade de beneficiamento. A base salarial utilizada foi a divulgada pelo Datafolha Instituto de Pesquisas (2014), no mês de agosto, para indústria, que resultou num custo 
anual de $R \$ 283.920,00$ com salários. Os custos trabalhistas envolvidos foram de $R \$$ 150.000,00, tendo por base as despesas adicionais de FGTS, 13ํㅗário, INSS, 1/3 de férias e férias. Foram desprezados os custos de vale transporte, vale refeição, plano de saúde e outros benefícios. As despesas com contabilidade foram definidas em um salário mínimo de $\mathrm{R} \$ 724,00$ por mês ou $\mathrm{R} \$ 8.688,00$ ao ano.

A alíquota do Simples Nacional utilizada foi de $8,10 \%$. Isso em decorrência do faturamento bruto anual da unidade estudada, que ficou dentro da faixa de mais de $R \$$ 720 mil até $\mathrm{R} \$ 900$ mil. Conforme Lei Complementar nํ 139, de 2011, Tabela Simples Nacional para Indústria (BRASIL, 2011).

Para o cálculo de depreciação estimou-se em dez anos a vida útil das máquinas e equipamentos, ao passo que as mesmas serão utilizadas somente por nove anos, sendo que no último serão vendidas pelo valor residual de $R \$ 54.084,00$. Chegou-se ao valor residual descontando do valor inicial as depreciações contabilizadas durante os nove anos de duração do projeto. A estrutura física não foi considerada no cálculo devido suas características contábeis para inserção no cômputo, onde a taxa seria de somente $4 \%$ ao ano, levando, assim, 25 anos para depreciar-se totalmente. Como o projeto prevê uma duração máxima de nove anos com venda dos ativos no décimo ano, o valor esperado de valorização do imóvel no período será totalmente capaz de suprir a necessidade de provisionamento de valores para reposição das benfeitorias/obras. A fórmula utilizada para cálculo da depreciação é apresentada na eq. (1):

$$
\text { Depreciação }=\frac{(\text { valor inicial }- \text { valor residual })}{\text { vida útil }}
$$

A despesa com combustível foi calculada com base na autonomia média de 1,5 $\mathrm{km} \mathrm{L}^{-1}$ de diesel, com preço médio de $\mathrm{R} \$ 2,37$ por litro numa situação de utilização do caminhão durante 256 dias por ano com média de rodagem de $60 \mathrm{~km}$ diários, totalizando $\mathrm{R} \$ 24.269,00$ ao ano. Para estabelecimento do percurso percorrido levou-se em consideração a localização dos principais pontos consumidores de coco verde na cidade de São Luís, bem como a distância existente entre a zona urbana da cidade e o distrito industrial.

O consumo de energia elétrica foi estimado levando-se em consideração o tamanho das áreas cobertas que necessitam de iluminação artificial, a quantidade de motores elétricos e os equipamentos utilizados pela administração. O cálculo de megawatt-horas consumidos por ano foi realizado utilizando o simulador de consumo 
disponibilizado pela Rio Grande Energia (2014), uma empresa do grupo CPFL Energia. Os seguintes equipamentos foram utilizados na simulação: um aparelho de ar condicionado Split 30.000 btus; duas impressoras; dois computadores; 75 lâmpadas fluorescentes comuns de 40 watts; um motor elétrico 10,0 cv; um motor elétrico 5,0 cv; dois motores $0,5 \mathrm{cv}$ e um motor $15,0 \mathrm{cv}$. Os motores descritos são partes das máquinas utilizadas no processamento das cascas de coco verde. Com a simulação obteve-se um consumo anual de aproximadamente $63,68 \mathrm{Mwh}$. O preço do Mwh utilizado nos cálculos foi $\mathrm{R} \$ 348,00$, referente a 18 set. 2014 , conforme divulgado pelo Sistema FIRJAN (2014), resultando em um custo anual de $R \$ 22.159,00$.

Foi estimado em $1.300 \mathrm{~m}^{3}$ o consumo de água anualmente a um preço médio para indústria de $R \$ 6,45$ por $\mathrm{m}^{3}$, totalizando $R \$ 8.385,00$ ao ano. O preço médio utilizado tem por base o mês de setembro de 2014, conforme a Companhia de Saneamento Ambiental do Maranhão [CAEMA] (2014). Além disso, foi considerado um custo anual com materiais de segurança e de escritório, no valor de $R \$ 4.000,00$ e $R \$$ $2.000,00$, respectivamente.

\section{Preços de venda da fibra e do pó de coco}

Os valores utilizados como referência para os preços de venda das fibras de coco e pó de coco foram obtidos a partir do cálculo da média das dez ofertas melhores classificadas para cada produto no Alibaba ${ }^{2}$, maior site de e-commerce do mundo. Os preços, que são apresentados em dólares americanos, foram convertidos para reais, valendo-se da média simples da cotação da moeda americana entre setembro de 2013 e agosto de 2014, que ficou em $\mathrm{R} \$ 2,28$, conforme séries temporais do BACEN (2014). O valor médio obtido para uma tonelada de fibra de coco foi de $\mathrm{R} \$ 677,16$ e para o pó de coco foi de $R \$ 352,83$.

\section{Análise econômica}

A partir de um fluxo de caixa líquido foram aplicados os indicadores econômicos valor presente líquido [VPL], taxa interna de retorno [TIR] e payback descontado. Segundo Carr et al. (2010), estes indicadores estão entre os mais utilizados em diferentes setores econômicos. Além disso, foi realizada uma análise de sensibilidade.

\footnotetext{
${ }^{2}$ ALIBABA.COM. 2014. Products. Disponível em: http://www.alibaba.com/Agricultural-Waste_pid138. Acesso em: 20 ago. 2014.
} 
O VPL foi calculado a partir da subtração dos retornos líquidos dos fluxos de caixa durante a duração do projeto pelo valor presente do investimento, conforme Puccini (2011), eq. (2):

$$
V P L=\left[\sum_{t=1}^{n} \frac{F C_{t}}{(1+K)^{t}}\right]-\left[I_{0}+\sum_{t=1}^{n} \frac{I_{t}}{(1+K)^{t}}\right]
$$

onde, $\mathrm{FC}_{\mathrm{t}}$ : é o fluxo de caixa no período $\mathrm{t}$, com $\mathrm{t}$ variando de $1 \mathrm{a} \mathrm{n}$, com $\mathrm{n}$ igual a 10 anos; K: é taxa de desconto do projeto, representada pela taxa mínima de atratividade $[T M A] ; I_{0}$ : é o investimento inicial no momento zero; e, $I_{t}$ : é valor do investimento previsto em cada período.

A TMA anual foi definida em 7,13\% em função da escolha de utilização de recursos próprios para financiar o projeto. Chegou-se ao resultado a partir da subtração da taxa Selic média mensal anualizada entre janeiro de 2.000 e agosto de 2014 $(14,04 \%)$ pela média mensal anualizada do Índice Nacional de Preços ao Consumidor Amplo [IPCA] do mesmo período (6,45\%). Os dados foram obtidos do BACEN (2014).

A TIR é a taxa de juros que iguala as entradas e saídas de recursos do projeto em algum determinado momento no tempo, ou seja, é a taxa de juros que zera o valor presente de um fluxo de caixa, conforme Puccini (2011), eq. (3):

$$
I_{0}+\sum_{t=1}^{n} \frac{I_{t}}{(1+k)^{t}}=\sum_{t=1}^{n} \frac{F C_{t}}{(1+k)^{t}}
$$

onde, k: é a taxa de rentabilidade equivalente periódica resultante (TIR).

O payback descontado é o tempo necessário para que se recupere o investimento inicial do projeto a partir das entradas de fluxo de caixa, considerando o efeito do tempo no dinheiro, ou seja, antes de calcular-se o payback descontado é preciso computar o valor presente dos fluxos de caixa (Weston e Brigham, 2000).

A sensibilidade do projeto foi testada a partir da fixação de uma variação positiva e negativa de $10 \%$ no preço dos produtos vendidos, bem como no valor dos investimentos iniciais. Com isso buscou-se avaliar os impactos das alterações nos indicadores de análise financeira testados. 


\section{Resultados e Discussões}

\section{Aspectos técnicos do processamento}

O processamento de $1.200 \operatorname{cocos} \mathrm{h}^{-1}$ está abaixo do indicado nos estudos elaborados por Mattos et al. (2014), que orienta a reciclagem mínima de 1.500 cocos h ${ }^{1}$ para viabilizar o empreendimento. No estudo daquela instituição, não foi considerado a utilização da capacidade instalada [UCI] da indústria. O presente trabalho demonstrou que mesmo com a proposta de redução da quantidade de matéria prima beneficiada por hora e com a aplicação do índice de $\mathrm{UCl}$ de $83,10 \%$, foi possível obter-se uma quantidade significativa de produtos: 515 toneladas de fibras e 1.201 toneladas de pó, anualmente. Com a redução do processamento, diminuiu-se a necessidade do espaço físico em $230 \mathrm{~m}^{2}$, além de aumentar a vida útil das máquinas e restringir as despesas com manutenção.

\section{Investimento inicial}

O investimento inicial previsto do projeto foi de $R \$ 835.065,00$, sendo que aproximadamente $66 \%$ desse valor referem-se ao dispêndio com obras e com a aquisição do terreno. Esse gasto poderia ser reduzido, inicialmente, em $R \$ 161.840,00$, caso não fosse realizada a aquisição do terreno para instalação da empresa e sim seu arrendamento ou aluguel. A opção de não adquirir o terreno elevaria no momento inicial do projeto os retornos dos fluxos de caixa em virtude da redução do investimento no ano zero. No entanto, a opção escolhida mostrou-se extremamente vantajosa diante do preço ofertado pelo proprietário, que foi de $\mathrm{R} \$ 80,00$ por $\mathrm{m}^{2}$. O custo anual da aquisição do terreno, considerando-se os dez anos de duração do projeto, ficou em $\mathrm{R} \$ 16.184,00$ ou $\mathrm{R} \$ 1.349,00$ mensais.

\section{Fluxo de caixa}

Com a produção anual de 515 t de fibras e 1.201 t de pó, pode-se obter receita total no primeiro ano do fluxo de caixa da ordem de $R \$ 772.486,00$, isso considerando o preço de venda dos produtos com base nas negociações praticadas no mercado internacional.

Após a redução das despesas operacionais, que somaram $R \$ 503.821,00$, o resultado operacional do primeiro ano ficou em $R \$ 268.665,00$. A análise dos demais períodos é dispensável em decorrência da utilização de taxas de juros reais para cálculo dos indicadores financeiros. A utilização de uma TMA baseada em taxas reais 
(descontando a inflação do período) possibilitou a fixação das quantidades produzidas e dos preços dos produtos vendidos durante todo o período de análise do projeto.

Após atenuação da depreciação e do simples nacional, o fluxo de caixa projetado para todos os períodos ficou em $R \$ 180.475,00$, a exceção do ano 10 , que atingiu $R \$$ $54.084,00$, em virtude da venda de ativos pelo valor residual. Não foram observadas no referido período outras receitas em função das características do projeto, que previa duração máxima de dez anos, posto que no último ano aconteça a eliminação do mesmo, conforme apresentado na Tabela 3. Ressalta-se que a consideração da depreciação de máquinas e equipamentos como saída no fluxo de caixa, apesar de penalizar o projeto, compensa despesas com manutenção e outras possíveis despesas não consideradas.

Tabela 3. Fluxo de caixa resumido de um projeto de implementação de uma unidade de processamento de cascas de coco verde em São Luís, Maranhão

\begin{tabular}{|c|c|c|c|c|c|c|}
\hline Descrição & Ano 0 & Ano 1 & Ano 2 & $\cdots$ & Ano 9 & Ano 10 \\
\hline & & & ---- & & 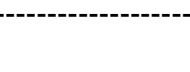 & ---------- \\
\hline \multicolumn{7}{|l|}{ Entradas } \\
\hline Venda de ativos & & & & & & $54.084,00$ \\
\hline $\begin{array}{c}\text { Venda de Fibras de } \\
\text { Coco }\end{array}$ & & $348.737,00$ & $348.737,00$ & $\cdots$ & $348.737,00$ & \\
\hline Venda de Pó de Coco & & $423.749,00$ & $423.749,00$ & $\cdots$ & $423.749,00$ & \\
\hline Receita total & & $772.486,00$ & $772.486,00$ & $\cdots$ & $772.486,00$ & $54.084,00$ \\
\hline \multicolumn{7}{|l|}{ Saídas } \\
\hline Despesa operacional & & $\begin{array}{c}(503.821,00 \\
)\end{array}$ & $\begin{array}{c}(503.821,00 \\
)\end{array}$ & $\cdots$ & $\begin{array}{c}(503.821,00 \\
)\end{array}$ & \\
\hline Depreciação & & $(25.619,00)$ & $(25.619,00)$ & $\cdots$ & $(25.619,00)$ & \\
\hline Simples nacional & & $(62.571,00)$ & $(62.571,00)$ & $\ldots$ & $(62.571,00)$ & \\
\hline $\begin{array}{l}\text { Investimento inicial } \\
\text { Total de saídas }\end{array}$ & $\begin{array}{c}(835.065,00 \\
) \\
(835.065,00 \\
)\end{array}$ & $\begin{array}{c}(592.011,00 \\
)\end{array}$ & $\begin{array}{c}(592.011,00 \\
)\end{array}$ & $\cdots$ & $\begin{array}{c}(592.011,00 \\
)\end{array}$ & \\
\hline Fluxo de caixa líquido & $\begin{array}{c}(835.065,00 \\
)\end{array}$ & $180.475,00$ & $180.475,00$ & $\cdots$ & $180.475,00$ & $54.084,00$ \\
\hline
\end{tabular}

Fonte: Elaborado pelo autor

\section{Análise econômica}

Os parâmetros escolhidos para o cenário base de elaboração das análises econômicas do projeto refletem a realidade observada na cidade de São Luís, em que este projeto, obteve um VPL (7,13\%) de R $\$ 361.458,00$, TIR de $16 \%$ e payback descontado de cinco anos e dez meses. Esses indicadores sugerem que o projeto é 
financeiramente atrativo e deve ser aceito, uma vez que se alcançou uma TIR maior que a TMA, bem como VPL positivo e payback inferior ao prazo de duração do projeto. É importante destacar que o prazo de duração do projeto foi definido em dez anos, ou seja, houve a necessidade de aguardar-se aproximadamente $60 \%$ do tempo total previsto do projeto para reaver o investimento realizado no momento inicial. Dessa forma, tornou-se indispensável a análise conjunta dos indicadores financeiros para aceitação do projeto no cenário base.

\section{Análise de sensibilidade}

Ao simular-se um cenário onde o investimento inicial sofreu alteração positiva e negativa de $10 \%$, verificou-se que em ambos os casos o projeto ainda se mostrou financeiramente atrativo. Importante destacar que mesmo com a simulação de elevação do investimento para $R \$ 918.572,00$, a TIR manteve-se superior a TMA base do projeto, definida em 7,13\%, demonstrando dessa forma que mesmo com uma variação considerável no valor do investimento inicial o projeto mostrou-se viável (Tabela 4).

Tabela 4. Alteração do investimento inicial e preços de venda da fibra e pó de coco para cálculo da sensibilidade dos indicadores econômicos

\begin{tabular}{|c|c|c|c|c|}
\hline Variável & Descrição & Cenário base & Redução de $10 \%$ & Acréscimo de $10 \%$ \\
\hline \multirow{4}{*}{ 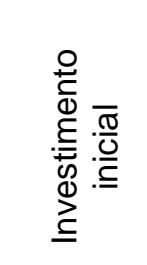 } & Investimento $(\mathrm{R} \$)$ & $835.064,00$ & $751.559,00$ & $918.572,00$ \\
\hline & $\operatorname{VPL}^{(1)}(\mathrm{R} \$)$ & $361.458,00$ & $444.965,00$ & $277.952,00$ \\
\hline & $\operatorname{TIR}^{(2)}(\%)$ & 16 & 19 & 14 \\
\hline & Payback & $\begin{array}{c}5 \text { anos e } 10 \\
\text { meses }\end{array}$ & 5 anos e um mês & 6 anos e 6 meses \\
\hline \multirow{5}{*}{$\begin{array}{l}\frac{\pi}{0} \\
\frac{0}{10} \\
0 \\
0 \\
0 \\
0 \\
0 \\
\Phi \\
\frac{1}{2}\end{array}$} & Fibra de coco $(R \$)$ & 677,16 & 609,44 & 744,88 \\
\hline & Pó de coco (R\$) & 352,83 & 317,55 & 388,11 \\
\hline & $\operatorname{VPL}^{(1)}(\mathrm{R} \$)$ & $361.458,00$ & $-98.521,00$ & $821.431,00$ \\
\hline & $\operatorname{TIR}^{(2)}(\%)$ & 16 & 4 & 27 \\
\hline & Payback & $\begin{array}{c}5 \text { anos e } 10 \\
\text { meses }\end{array}$ & $>10$ anos & $\begin{array}{c}3 \text { anos e } 11 \\
\text { meses }\end{array}$ \\
\hline
\end{tabular}

Nota: (1) VPL: Valor Presente Líquido; (2) TIR: Taxa Interna de Retorno

Fonte: Elaborado pelo autor

Ao projetar-se uma alteração de $10 \%$ no preço dos produtos vendidos, pode-se verificar que o projeto se mostrou extremamente sensível às alterações promovidas, tendo uma variação acentuada tanto com a elevação do preço, onde o VPL atingiu R\$ 
821.431,00, quanto com a redução, onde o projeto apresentou-se inviável do ponto de vista financeiro com VPL negativo de $\mathrm{R} \$ 98.521,00$ e TIR inferior a TMA (Tabela 4).

\section{Conclusões}

A implantação de uma unidade de processamento de cascas de coco verde em São Luís apresenta-se promissora e economicamente viável no cenário base analisado.

O projeto é extremamente sensível às variações de preço dos produtos vendidos. Ao reduzir-se em $10 \%$ o preço da fibra e pó de coco, as análises econômicas indicaram que o projeto deve ser rejeitado. Porém em todos os demais cenários analisados o projeto apresentou-se viável.

É importante frisar que o presente trabalho conjectura as possibilidades de comercialização somente dos produtos básicos extraídos das cascas do coco verde, sendo possível outros trabalhos avaliarem a viabilidade de desenvolvimento de produtos mais complexos e de maior valor agregado, tais como os produtos para jardinagem, bancos de veículos, mantas de contenção de erosões, substrato agrícola, chapas de fibras de coco, cordas, vassouras, capachos, entre outros.

\section{Referências}

Banco Central do Brasil [BACEN]. 2014. SGS: sistema gerenciador de séries temporais. Disponível em:

<https://www3.bcb.gov.br/sgspub/localizarseries/localizarSeries.do?method=prepararT elaLocalizarSeries>. Acesso em: 20 ago. 2014.

BRASIL. 2011. Lei n. 139, de 10 de novembro de 2011. Altera dispositivos da lei complementar n. 123, de 14 de dezembro de 2006, e dá outras providências. Diário Oficial, Brasília, 11 nov. 2011. Seção 1, p. 1-6.

Carr, C.; Kolehmainen, K.; Mitchell, F. 2010. Strategic investment decision making practices: a contextual approach. Management Accounting Research 21: 167-184.

Companhia de Saneamento Ambiental do Maranhão [CAEMA]. 2014. Preço da água para indústria. Disponível em:

<http://gsan.caema.ma.gov.br:8080/gsan/exibirServicosPortalCaemaAction.do>. Acesso em: 20 ago. 2014.

Cuenca, M.A.G. 2007. A cultura do coqueiro. Embrapa Tabuleiros Costeiros.

Disponível em:

<http://sistemasdeproducao.cnptia.embrapa.br/FontesHTML/Coco/ACulturadoCoqueir o/importancia.htm>. Acesso em: 25 ago. 2014.

DATAFOLHA INSTITUTO DE PESQUISAS. 2014. Salários: Consulta por segmento. Disponível em:

<http://datafolha1.folha.com.br/empregos/segmentos?reference_date=2014-08- 
11\&segment_id=3\&find=Enviar $>$. Acesso em: 20 ago. 2014.

Foale, M.; Harries, H. 2009. Farm and forestry production and marketing profile for coconut (Cocos nucifera). Permanent Agriculture Resources. Disponível em:

$<$ http://agroforestry.net/images/pdfs/Coconut_specialty_crop.pdf>. Acesso em: 25 ago. 2014.

Food and Agriculture Organization of the United Nations [FAO]. 2013. FAOSTAT:

Production. Disponível em: <http://faostat3.fao.org/faostat-

gateway/go/to/browse/Q/*/E>. Acesso em: 20 ago. 2014.

Fundação Instituto de Pesquisas Econômicas [FIPE]. 2014. Preço médio de veículos: caminhões. Disponível em:

<http://www.fipe.org.br/web/index.asp?aspx=/web/indices/veiculos/introducao.aspx>.

Acesso em: 20 ago. 2014.

Instituto Brasileiro de Geografia e Estatística [IBGE]. 2014. Produção agrícola municipal. Disponível em: <http://www.sidra.ibge.gov.br>. Acesso em: 20 ago. 2014.

Mattos, A.L.A.; Rosa, M.F.; Crisóstomo, L.A.; Figueiredo, M.C.B.; Veras, L.G.C. 2011. Processamento da casca de coco verde para a produção de pó e fibra. Journal of the InterAmerican Society for Tropical Horticulture 53: 85-88.

Mattos, A.L.A.; Rosa, M.F.; Crisóstomo, L.A.; Bezerra, F.C.; Correia, D.; Veras, L.G.C. 2014. Beneficiamento da casca de coco verde. Embrapa Agroindústria Tropical. Disponível em: <http://www.ceinfo.cnpat.embrapa.br/arquivos/artigo_3830.pdf>. Acesso em: 25 ago. 2014.

Puccini, E.C. 2011. Matemática financeira e análise de investimentos. UFSC, Florianópolis, Santa Catarina, Brasil.

RIO GRANDE ENERGIA. 2014. Simulador de consumo. Disponível em: $<$ http://www.rgers.com.br/ServicosRGE/servicosonline/simuladorconsumo/calculo_consumo.asp>. Acesso em: 20 ago. 2014.

Sindicato Nacional dos Produtores de Coco do Brasil [SINDCOCO]. 2014. Palestra fenacoco. Disponível em: <http://www.sindcoco.com.br>. Acesso em: 20 ago. 2014.

SISTEMA FIRJAN. 2014. Quanto custa a energia elétrica para a indústria no Brasil? Disponível em: <http://www.quantocustaenergia.com.br>. Acesso em: 20 ago. 2014.

Weston, J.F.; Brigham, E.F. 2000. Fundamentos da administração financeira. MAKRON Books, São Paulo, São Paulo, Brasil. 